\title{
Mode identification using the exoplanetary camera
}

\author{
R. Garrido ${ }^{1}$, A. Moya ${ }^{1}$, M. J.Goupil ${ }^{2}$, C. Barban ${ }^{2}$, C. van't Veer-Menneret ${ }^{2}$, \\ F. Kupka ${ }^{3}$ and U. Heiter ${ }^{3}$ \\ 1.-Instituto de Astrofisica de Anadalucia, Apdo. 3004, Granada, Spain \\ 2.-DASGAL, UMR CNRS 8633, Observatoire de Paris-Meudon, France \\ 3.-Institut für Astronomie, Universität Wien, Austria
}

\begin{abstract}
Numerical simulations are presented, which show that color information, as supplied by the exoplanetary camera of COROT, can be relevant to discriminate among several spherical degrees $\ell$ of the non-radial oscillations of $\delta$ Scuti stars.
\end{abstract}

\section{Introduction}

Multiperiodic $\delta$ Scuti stars show power spectra indicating that they pulsate in a mixing of radial and non-radial modes. Whereas period ratios can be used as an indication of the radial order $n$ for radial modes they are not useful for the non-radial ones. Rotation and evolution -the so called "avoided crossing" phenomenon- complicate even more any mode identification. Spectroscopy through line profile variations- and photometry -through the use of amplitude ratios and phase differences of different colors- have been widely utilized as techniques for mode identification. Here we will focus on the capability of the explanetary camera in COROT to supply color information which can be relevant for mode identification.

\section{Numerical simulations}

In front of the exoplanetary camera a bi-prism device will supply colored spotted images which will give three colors. These colors will depend on the spectral type of the considered star and on the position over the CCD plane. The full spot will be divided into three parts called "blue", "green" and "red", containing $20 \%, 20 \%$ and $60 \%$ respectively of the total flux. 


\section{b/g, 7500K, 4, .035}

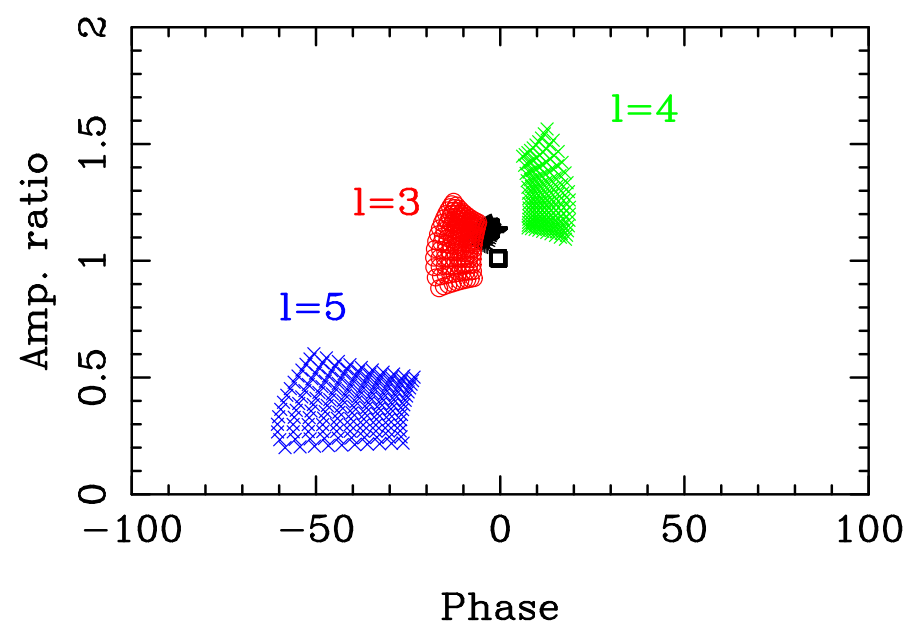

Figure 1: Amplitude ratio vs phase differences between "blue" and "green" bands of the exoplanetary camera of COROT for a model atmosphere of $T=7500 \mathrm{~K}$, $\log g=4.0$ and periods close to a pulsation cosntant of $Q=0.035 d$. Regions for every $\ell$ value contain predictions for phase lags between 90 and 135 degrees and values of $R$ between 0.25 and 1.00 .

The Kurucz model atmospheres have been improved in the way explained in detail in Heiter et al. (2002), basically by a better treatment of the convection and a larger number of shells in order to obtain good derivatives with respect to temperature and gravity.

The linearised formula given by Watson (1988) has been used to compute the diagrams shown in Figure 1 and 2. Regions for different $\ell$ have been evaluated for an improved model atmosphere of $T=7500 \mathrm{~K}, \log g=4$, Pop I and a period region around the fundamental radial mode -pulsation constant $Q=0.035 d$-following the paper by Garrido et al. (1990). Basically the predictions are based on calculations assuming a phase lag -angle between temperature and radius variations- between 90 and 135 degrees and an $R$ value -a parameter measuring the non-adiabaticity of the atmosphere- between 0.25 and 1.00 .

From these figures we can see a clear separation for high $\ell$ orders, giving very different regions in the amplitude ratio vs phase differences plane. For the lowest $\ell$ orders this separation is less evident but we expect that the accuracy at which COROT will supply light curves allows a precision for amplitudes and 
b/g, 7500K, 4, .035

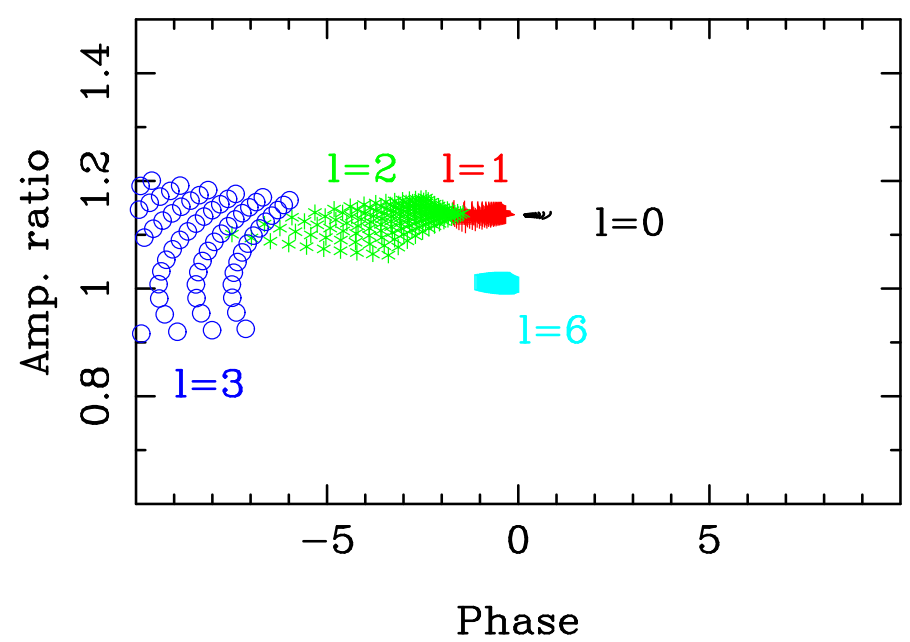

Figure 2: Same as figure 1 but for the central region.

phases sufficient to discriminate among several $\ell$ orders and hence to help doing asteroseismology of these stars.

\section{References}

Garrido, R., Garcia-Lobo, E., Rodriguez, E. 1990, A\&A 234, 262

Heiter, H., et al. 2002, in preparation

Watson, R. D. 1988, Ap\&SS 140, 255 